\title{
Gestational choriocarcinoma with residual lung tumor after completing treatment: a case report
}

Sara Centonze, $\mathrm{MD}^{\mathrm{a}}$, Elisena Franzese, $\mathrm{MD}^{\mathrm{a}, \star}$, Anna Diana, MD ${ }^{\mathrm{a}}$, Sandro Pignata, $\mathrm{MD}^{\mathrm{b}}$, Fortunato Ciardiello, MD , Michele Orditura, MD

\begin{abstract}
Introduction: Choriocarcinoma (CCA) is a malignant pregnancy-related tumor that originates from cytotrophoblast and syncytiotrophoblast cells without villi. CCA is the most metastatic form among gestational trophoblastic neoplasia and the diagnosis is often in advanted stage.

Patient concerns: In this report, we present a rare case of CCA with lung metastasis after term pregnancy in a 41 -year-old woman at 45 days after the birth of a healthy baby and discuss management of residual metastasis after completing treatment.

Diagnosis: The diagnosis of CCA can be based on the following criteria: a human chorionic gonadotropin (hCG) plateau for at least 4 values over 3 weeks, an hCG increase of 10\% or greater for at least 3 values over 2 weeks, hCG persistence for 6 months or more after molar pregnancy evacuation, histopathologic diagnosis of choriocarcinoma and presence of metastatic disease.

Intervention and outcomes: High risk patients must be treated with multiagent chemotherapy (EMA-CO schedule). The complete remission rates ranges from $69 \%$ to $86 \%$.

Conclusion: Residual lesions after chemotherapy are often the result of necrosis or fibrosis. In our clinical practice will avoid the risks deriving from excessive anticancer treatment in these patients, while favoring a strict follow-up strategy to monitor disease behavior in time.
\end{abstract}

Keywords: Choriocarcinoma, Gestational trophoblastic neoplasia, Residual tumor, Case report

Gestational trophoblastic neoplasia (GTN) includes a group of malignant pregnancy-related tumors such as invasive mole, choriocarcinoma (CCA), placental site trophoblastic tumor and epithelioid trophoblastic tumor. Among them, CCA is the most metastatic form with varied incidence worldwide. Previous complete hydatiforme mole (a 100-fold increased risk), advanced maternal age ( $40 \mathrm{y})$, family history of molar pregnancy, ethnicity, long-term oral contraceptive use are the main risk factors ${ }^{[1]}$. About $50 \%$ of cases arise from hydatiform mole, while the remaining $50 \%$ occur following abortion, tubal pregnancy, and term or preterm gestation ${ }^{[1]}$. CCA originates from cytotrophoblast and syncytiotrophoblast cells without villi and produces

\footnotetext{
a Department of Precision Medicine, Division of Medical Oncology, School of Medicine, "Luigi Vanvitelli" University of Campania, Naples, Italy and 'bstituto Nazionale per lo Studio e la Cura dei Tumori "Fondazione G. Pascale," IRCCS

This manuscript has been peer reviewed.
}

Sponsorships or competing interests that may be relevant to content are disclosed at the end of this article.

${ }^{*}$ Corresponding author. Address: Istituto Nazionale per lo Studio e la Cura dei Tumori "Fondazione G. Pascale," IRCCS, Naples 80131, Italy. Tel: +39 0815666713. E-mail address: elifranzese@hotmail.it (E. Franzese).

Copyright @ 2020 The Authors. Published by Wolters Kluwer Health, Inc. on behalf of IJS Publishing Group Ltd. This is an open-access article distributed under the terms of the Creative Commons Attribution-Non Commercial-No Derivatives License 4.0 (CCBY-NC-ND), where it is permissible to download and share the work provided it is properly cited. The work cannot be changed in any way or used commercially without permission from the journal.

International Journal of Surgery Oncology (2020) 5:e107

Received 16 September 2020; Accepted 23 October 2020

Published online 6 January 2021

http://dx.doi.org/10.1097//J9.0000000000000107 human chorionic gonadotrop (hCG) ${ }^{[1]}$. Histologic examination shows abnormal trophoblastic hyperplasia and anaplasia, absence of chorionic villi, hemorrhage and necrosis with direct invasion into the myometrium, and vascular invasion resulting in spread to distant sites, most commonly the lungs, brain, liver, pelvis and vagina, kidney, intestine, and spleen ${ }^{[2]}$. Diagnosing intraplacental CCA, which appears as a focal neoplastic proliferation of the chorionic villous trophoblast in an otherwise normal third trimester placenta ${ }^{[3]}$, is difficult owing to its rarity. Immunohistochemically, the neoplastic syncytiotrophoblastic cells show a strong and diffuse positivity for hCG and HSD3B1, Mel-CAM, HLA-G, and MUC-4, CK,AE1/AE3. Furthermore, a high Ki-67 labeling index (>90\%) is typically observed ${ }^{[2]}$. CCA produces high levels of angiogenic growth factors leading to remodelling of uterine vasculature with formation of fragile vessels, thus resulting in irregular vaginal bleeding ${ }^{[4]}$. The tendency to metastasize early, often with hemorrhagic lesions, explains why first symptoms are frequently related to brain hemorrhage, hemoptysis, gastrointestinal bleeding ${ }^{[5]}$. Furthermore, trophoblastic emboli may cause pulmonary arterial occlusion and subsequent right-heart strain. Patients with suspected CCA should receive pelvic ultrasonography to look for retained trophoblastic tissue appearing as an echogenic uterine mass with areas of necrosis and hemorrhage and hypervascular pattern on Doppler analysis. Chest radiography or computed tomography are required to detect lung metastases. Brain magnetic resonance imaging or lumbar puncture may be used to search for occult cerebral or meningeal disease. Moreover, pelvic magnetic resonance may be used to assess extrauterine invasion. Therefore, according to the International Federation of Gynecology and Obstetrics (FIGO), the diagnosis of CCA can be based on the following criteria: an hCG plateau for at least 4 values over 3 weeks, 
an hCG increase of $10 \%$ or greater for at least 3 values over 2 weeks, hCG persistence for 6 months or more after molar pregnancy evacuation, histopathologic diagnosis of choriocarcinoma and presence of metastatic disease ${ }^{[6]}$.

In this report, we present a rare case of CCA with lung metastasis after term pregnancy in a 41-year-old woman at 45 days after the birth of a healthy baby and discuss management of residual metastasis after completing treatment.

\section{Case presentation}

In September 2018, a 41-year-old woman was referred to our cancer center with a diagnosis of postpartum CCA. There was no family history of CCA or relevant genetic information.

In July 2018, the patient had successfully delivered a term pregnancy without complications. The placenta was expelled but not sent for pathology review. After childbirth, on postpartum day 10 , she experienced small amounts of vaginal bleeding that increased over the next few days. On postpartum day 40, the patient was transferred to the gynecological clinic. Physical examination showed an enlarged uterus $(123 \times 62 \mathrm{~mm})$. Ultrasonography revealed an echogenic uterine mass $(40 \times 20 \times 1.5 \mathrm{~mm})$ with areas of necrosis and hemorrhage and hypervascular pattern on Doppler analysis. The blood hCG level was 500,000 mIU/mL. Compute tomography (CT) scan revealed solid nodular lesions (Fig. 1A) $(>8)$ in both lungs, ranging from 10 to $22 \mathrm{~mm}$ in diameter. There was no evidence of brain or liver disease. In October 2018, the patient underwent biopsy of the uterine mass that confirmed the diagnosis of postpartum CCA. The patient was classified as having high-risk disease (stage III, score 9) based on FIGO staging and modified WHO risk-factor scoring system for gestational trophoblastic neoplasia. Therefore, first-line treatment with EMA-CO chemotherapy was given [Day 1: actinomycin-D 0.5 $\mathrm{mg}$ iv, etoposide $100 \mathrm{mg} / \mathrm{m}^{2}$ iv, methotrexate $300 \mathrm{mg} / \mathrm{m}^{2}$ iv. Day 2: actinomycin-D $0.5 \mathrm{mg}$ iv, etoposide $100 \mathrm{mg} / \mathrm{m}^{2}$ iv, folinic acid $15 \mathrm{mg}$ post 12 hourly for 4 doses. Day 8 : vincristine $0.8 \mathrm{mg} / \mathrm{m}^{2}$ (maximum $2 \mathrm{mg}$ ), cyclophosphamide $600 \mathrm{mg} / \mathrm{m}^{2}$ ], starting October 2018. Unfortunately, after day 8 of first cycle, the patient developed severe abdominal pain and bowel obstruction caused by a volvulus, complicated by severe intestinal necrosis. She underwent bowel resection and complete hystero-annessectomy as emergency surgery. The procedure was performed by resident surgeon.

The blood hCG level after surgery was $200,000 \mathrm{mIU} / \mathrm{mL}$. Because of the need to clear an abdominal abscess, the patient could restart chemotherapy late in December 2018 and underwent 8 cycles, with a modified regimen excluding vincristine. The treatment was well-tolerated, the main adverse events being G1 neutropenia and anemia and no dose reduction was deemed necessary.

In March 2019, the CT scan showed a partial response (PR) to treatment (reduction of lung lesions); normal hCG levels (3 mIU/ $\mathrm{mL}$ ) were observed. After discussing with the patient, 4 more cycles were administered. In May 2019, the CT scan revealed a further lung response, with lower blood hCG levels $(<0.05 \mathrm{mIU} / \mathrm{mL})$.
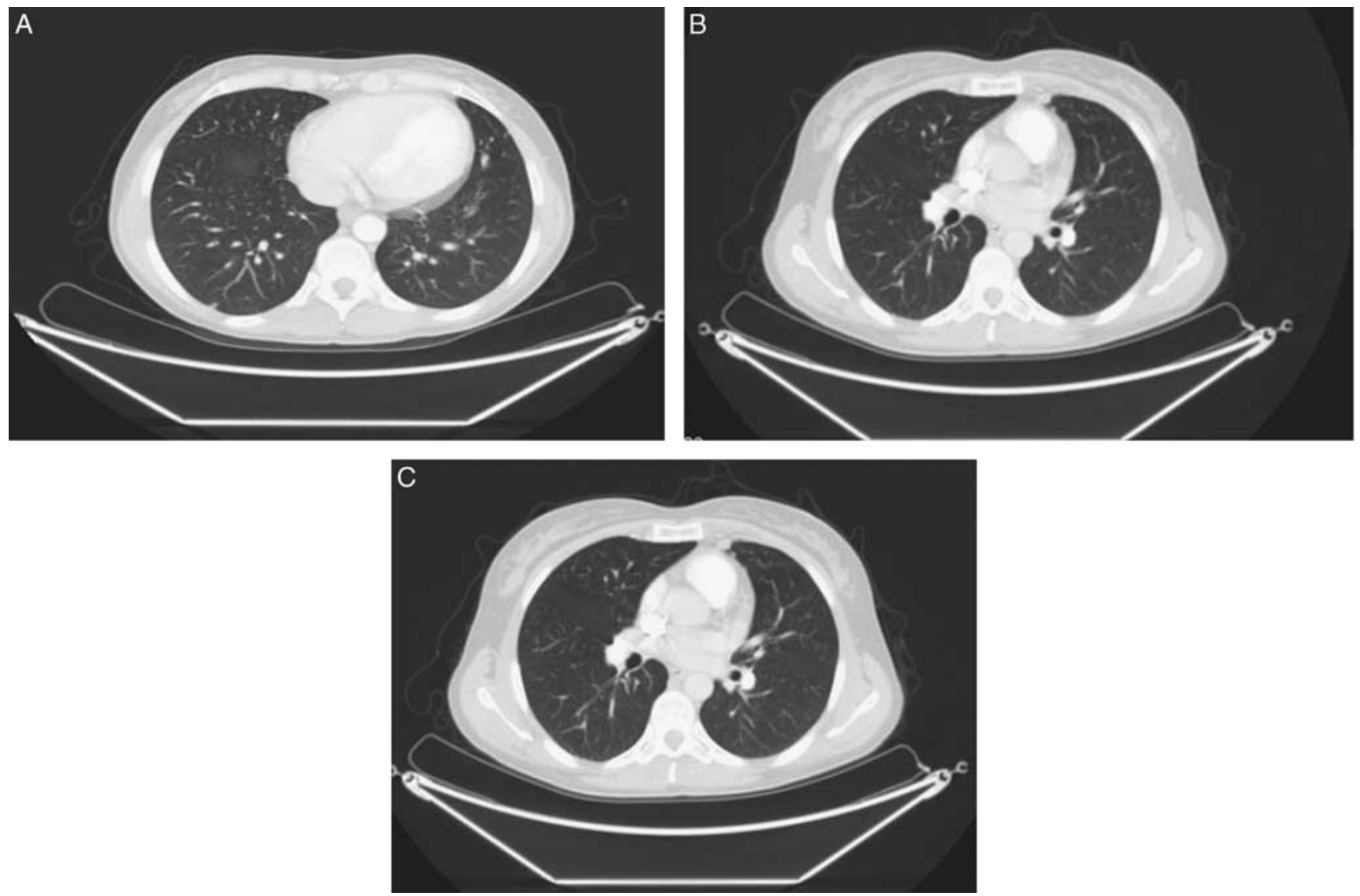

Figure 1. Comparison of pulmonary lesions. A, Lung computed tomography before chemotherapy. B, Lung computed tomography 3 months after chemotherapy. $\mathrm{C}$, Lung computed tomography 5 months after chemotherapy. 
Chemotherapy was stopped and a short-term follow-up was begun, with the patient undergoing a CT scan and assessment of hCG blood levels every 3 months. At the latest follow-up, in February 2020, the CT scan detected a further size reduction of pulmonary lesions (Figs. 1B, C) (maximum diameter $2 \mathrm{~mm}$ ), while blood hCG value was $1 \mathrm{mIU} / \mathrm{mL}$.

\section{Discussion}

The treatment of choice for gestational CCA depends on the combined anatomic FIGO staging and modified WHO risk-factor scoring system, which attribute the risk a patient has of developing chemoresistance ${ }^{[7]}$.

A WHO score $<6$ and FIGO stage I-II-III identify women at low risk amenable to single-agent chemotherapy, with cure rates between $80 \%$ and $90 \%$. On the other hand, patients with a WHO score $>6$ and FIGO stage II-III-IV are classified as being at high risk; therefore, multiagent chemotherapy with adjuvant radiation and/or surgery are required to achieve similar healing rates.

Many combination regimens have been developed over the years: MAC (MTX, actinomycin D and cyclophosphamide or chlorambucil), CHAMOCA (cyclophosphamide, hydroxyurea, actinomycin D, MTX, vincristine and doxorubicin), EMACP (etoposide, MTX, actinomycin D, cyclophosphamide and cisplatin); of these, the latter one has been designed so as to be repeated with a short interval between courses as well as to cause less myelosuppression. In 1979, EMA/CO chemotherapy (etoposide, MTX and actinomycin D, alternating with cyclophosphamide and vincristine) was introduced; currently, it is the most widely accepted initial treatment for high-risk metastatic GTN, with complete remission rates ranging from $69 \%$ to $86 \%{ }^{[8]}$. This regimen is generally well tolerated with few acute and chronic toxicities, including alopecia, nausea, reversible neurotoxicity, and myelosuppression. EMA/CO has also been compared with the EMACP regimen, which was not found to be more effective. However, it has not been rigorously compared with other combinations such as MAC or FAV in randomized trials ${ }^{[9]}$.

It is strongly recommended the use of induction low-dose EP in all patients with a high disease burden at presentation, that is, with a total FIGO prognostic score $>12$ and an hCG level $>1,000,000 \mathrm{IU} / \mathrm{L}^{[10]}$. These features are associated with early death due to development of such severe complications as hemorrhagic metastases, infection, multisystem organ failure, or tumor lysis syndrome. According to international guidelines, it is mandatory to continue chemotherapy for high-risk disease for at least 2-3 courses after the first normal hCG has been obtained, in order to reduce the likelihood of relapse ${ }^{[11]}$.

Therefore, after treatment, complete response $(\mathrm{CR})$ is defined when 3 consecutive normal values of hCG have been obtained and metastasis have disappeared, while a PR occurs when the hCGtiter has declined to $50 \%$ of its original level and the size of metastasis has been shown to have decreased.

However, some patients may have residual metastases at the end of chemotherapy despite a normal hCG titer. This condition, as reported in our case report, raises the question of whether to continue the treatment because only a PR has been obtained based on the residual disease on imaging or to interrupt it because of biochemical remission.

Junjun et $\mathrm{al}^{[12]}$ retrospectively analyzed the prognosis of 112 gestational GTN patients (44\% with CCA diagnosis) who presented with residual lung tumor and a normal hCG level after completing EMA/CO or EMA/EP treatment. Their residual tumor size remained constant even after administering additional cycles $(0-8)$ of chemotherapy. Furthermore, 13 pulmonary lobectomies were performed and histologic results showed necrotic and hemorrhagic tissues in 7 non-drug-resistant cases and in 1 drugresistant case, while trophoblastic tumor cells persisted in the remaining 5 drug-resistant cases. During follow-up, 83 patients $(63 \%)$ did not show significant changes in their residual metastatic disease, $46(30 \%)$ experienced a further reduction or disappearance of metastases, while the remaining $6(4 \%)$ patients had progression disease (PD), with hCG levels going up 6-8 months after treatment completion. The PD rate $(4 \%)$ of these patients was compared with the recurrence rate $(3.4 \%)$ of $901 \mathrm{CR}$ patients treated in the same institution during the same period. No significant statistical difference was observed. Therefore, the authors concluded that patients achieving normal $\beta$-hCG titers after treatment in spite of residual lung lesions, even in the case of additional courses of chemotherapy, should be considered as CR patients rather than PR patients. The residual lesions are most likely the result of necrosis or fibrosis. Incorporating these conclusions in our clinical practice will avoid the risks deriving from excessive anticancer treatment in these patients, while favoring a strict follow-up strategy to monitor disease behavior in time.

\section{Statement}

The present work has been reported in line with the SCARE 2018 criteria: Agha RA, Borrelli MR, Farwana R, Koshy K, Fowler A, Orgill DP, For the SCARE Group. The SCARE 2018 Statement: Updating Consensus Surgical CAse REport (SCARE) Guidelines, International Journal of Surgery 2018;60:132-136.

Written informed consent was obtained from the patient for publication of this case report and accompanying images. A copy of the written consent is available for review by the Editor-inChief of this journal on request.

\section{Ethical approval}

Written informed consent for publication was obtained from the patient.

\section{Sources of funding}

None declared.

\section{Author contribution}

C.S. and F.E.: conceptualization. C.S. and D.A.: data curation. F.E., C.S., and O.M.: formal analysis. F.E., C.S., and P.S.: investigation. F.E., C.S., and O.M.: methodology. O.M.: project administration. F.E., C.S., and D.A.: resources. D.A. and C.S.: software. C.F., O.M., and P.S.: validation

\section{Conflicts of interest disclosure}

The authors declare that they have no financial conflict of interest with regard to the content of this report. 


\section{Research registration unique identifying number (UIN)}

Not applied.

\section{Guarantor}

Michele Orditura.

\section{References}

[1] Lurain JR. Gestational trophoblastic disease I: epidemiology, pathology, clinical presentation and diagnosis of gestational trophoblastic disease, and management of hydatidiform mole. Am J Obstet Gynecol 2010;203: 531-9.

[2] Hui P. Gestational trophoblastic tumors: a timely review of diagnostic pathology. Arch Pathol Lab Med 2019;143:65-74.

[3] Heller DS. Update on the pathology of gestational trophoblastic disease. Apmis 2018;126:647-54.

[4] Singh M, Kindelberger D, Nagymanyoki Z, et al. Vascular endothelial growth factors and their receptors and regulators in gestational trophoblastic diseases and normal placenta. J Reprod Med 2012;57:197-203.
[5] Zhang W, Liu B, Wu J, et al. Hemoptysis as primary manifestation in three women with choriocarcinoma with pulmonary metastasis: a case series. J Med Case Rep 2017;11:110.

[6] Seckl MJ, Sebire NJ, Berkowitz RS. Gestational trophoblastic disease. Lancet 2010;376:717-29.

[7] Ngan H, Bender H, Benedet JL, et al. FIGO Committee on Gynecologic Oncology. Gestational trophoblastic neoplasia, FIGO 2000 staging and classification. Int J Gynaecol Obstet 2003;83:157-77.

[8] Deng L, Yan X, Zhang J, et al. Combination chemotherapy for high-risk gestational trophoblastic tumour. Cochrane Database Syst Rev 2009;15: CD005196.

[9] Lybol C, Thomas MG, Blanken EA. Comparing cisplatin-based combination chemotherapy with EMA/CO chemotherapy for the treatment of high risk gestational trophoblastic neoplasia. European J Cancer 2013;49: $860-7$.

[10] Alifrangis C, Agarwal R, Short D, et al. EMA/CO for high-risk gestational trophoblastic neoplasia: good outcomes with induction low-dose etoposidecisplatin and genetic analysis. J Clin Oncol 2013;31:280-6.

[11] Seckl MJ, Sebire NJ, Fisher RA, et al. Gestational trophoblastic disease: ESMO Clinical Practice Guidelines for diagnosis, treatment and followup. Ann Oncol 2013;24(suppl 6):vi39-50.

[12] Junjun Y, Yang X, Xirun W. The prognosis of gestational trophoblastic neoplasia patient with residual lung tumor after completing treatment. Gynecologic Oncology 2006;103:479-82. 